\title{
Ergebnisse der Untersuchung reiner Naturweine des Jahres 1901.
}

\author{
Von \\ Karl Windisch.
}

Mitteilung aus der önochemischen Versuchsstation zu Geisenheima. Rh.

Während der Vorwinter 1900/01 mild und regnerisch war, trat nach Neujahr 1901 starke Kälte ein, unter der die Weinstöcke in manchen Gegenden erheblich litten. Von Frühjahrsfrösten blieben die Reben im Jahre 1901 völlig verschont. Die Witterung war während des Sommers der Entwickelung der Trauben sehr günstig. Leider trat in der ersten Hälfte des September bei feuchter, warmer Witterung eine allgemeine Fäulnis der halbreifen Trauben ein, die mit einer nur selten beobachteten Energie fortschritt. Um der durch die starke Fäulnis bewirkten Schädigung der Qualität und Menge der Trauben entgegenzuwirken, wurde schleunigst mit der Ernte begonnen und diese ohne Unterbrechung zu Ende geführt. Die in der zweiten Hälfte des Oktober eintretende warme, trockene Witterung kam nur noch den wenigen, am Stocke belassenen Trauben zu gute, wirkte hier aber ganz hervorragend günstig. $\mathrm{E}_{\mathrm{s}}$ war sehr interessant, festzustellen, wie von Tag zu Tag das Mostgewicht und der Zuckergehalt stiegen und die Säure abnahm; in einem Falle (Weinberge der Königlichen Domäne in Rüdesheim) stieg z. B. das Mostgewicht allmählich auf über $130^{\circ}$ Ochsle, während der Säuregehalt der Moste bis auf $0,4 \mathrm{~g}$ in $100 \mathrm{ccm}$ herabging, ein im Jahre 1901 im Rheingau ganz vereinzelter Fall. Die Kennzeichen des Jahrganges 1901 sind im allgemeinen niedriges Mostgewicht und hohe Säure der Moste; die 1901-er Weine sind dementsprechend verhältnismäßig dünn, alkoholarm und sauer. Den besten Maßstab für die Beurteilung der Qualität eines Jahrganges gibt der Vergleich mit den vorhergehenden Jahrgängen; dieser Vergleich ergibt, daß die Weine des Jahres 1900 denen des Jahres 1901 aus gleichen Lagen fast durchweg ïberlegen sind.

Im folgenden sind die Ergebnisse der im Jabre 1902 in der önochemischen Versuchsstation zu Geisenheim a. Rh. ausgeführten Untersuchungen naturreiner Jungweine des Jahres 1901 zusammengestellt. Die Untersuchungen wurden von den Assistenten der Versuchsstation, Dr. G. Broichsitter und Dr. Fr. Müller, ausgeführt.

Was die durch das Weingesetz bezw. durch die auf Grund dieses Gesetzes erlassene Ausführungsverordnung festgesetzten Grenzzahlen anbetrifft, so wurde die Grenzzahl für den Extraktgehalt der Weissweine $(1,6 \mathrm{~g}$ in $100 \mathrm{ccm})$ nur in e inem Falle unterschritten, bei No. 47 der Tabelle, einem Elsässer Weine, der nur 1,504 $\mathrm{g}$ Extrakt in $100 \mathrm{ccm}$ enthielt. Der Extraktgehalt des Weines No. 20, eines Naheweines aus Rieslingtrauben, lag nur wenig über der gesetzlichen Grenze $(1,611 \mathrm{~g}$ in $100 \mathrm{ccm}$ ). Die Grenzzahl für den Extraktgehalt nach Abzug der Gesamtsäure $(1,0 \mathrm{~g}$ in $100 \mathrm{ccm})$ wurde in drei Fällen, bei den Weinen No. 33 $(0,940 \mathrm{~g})$, No. $47(0,774 \mathrm{~g})$ und No. $48(0,789 \mathrm{~g})$, unterschritten; fünf andere Weine ergaben Werte, die nahe an der Grenze liegen, nämlich No. 12 mit 1,050 g, No. 20 mit 1,061 g, No. 22 mit $1,050 \mathrm{~g}$; No. 30 mit 1,043 g, No. 46 mit 1,004 g Extrakt nach Abzug der Gesamtsäure. Ganz ähnlich liegen die Verhältnisse bezüglich der

[Fortsetzung auf S. 302.] 


\section{Weif}

A. Rhein

\begin{tabular}{|c|c|c|c|c|c|c|c|c|c|c|c|}
\hline \multirow{3}{*}{ No } & \multirow{3}{*}{$\begin{array}{c}\text { Ge- } \\
\text { markung }\end{array}$} & \multirow{3}{*}{$\begin{array}{c}\text { Lage } \\
\text { (Gewann) }\end{array}$} & \multirow{3}{*}{ Bodenart } & \multirow{3}{*}{$\begin{array}{c}\text { Trauben- } \\
\text { sorte }\end{array}$} & \multirow{3}{*}{$\begin{array}{c}\text { Zeit } \\
\text { der Lese }\end{array}$} & \multicolumn{2}{|c|}{$\begin{array}{l}\text { Zusammen- } \\
\text { setzung des } \\
\text { Mostes }\end{array}$} & \multirow{3}{*}{$\begin{array}{c}\text { Spez. } \\
\text { Ge- } \\
\text { wieht } \\
\text { bei } \\
15^{0} 0 .\end{array}$} & \multirow[b]{2}{*}{$\underset{\text { koliol }}{\mathrm{Al-}}$} & \multirow{2}{*}{$\begin{array}{c}\text { Ge- } \\
\text { samt- } \\
\text { Ex- } \\
\text { trakt } 1)\end{array}$} & \multirow{2}{*}{$\begin{array}{l}\text { Mine- } \\
\text { ralbe- } \\
\text { stand- } \\
\text { teile }\end{array}$} \\
\hline & & & & & & \multirow{2}{*}{$\begin{array}{c}\text { Most- } \\
\text { Ge- } \\
\text { wicht } \\
\text { (Grade } \\
\text { Öchsle) }\end{array}$} & \multirow{2}{*}{$\begin{array}{c}\text { Sänre } \\
\text { (als } \\
\text { Wein- } \\
\text { saure } \\
\text { berech- } \\
\text { net) } \\
\text { g in } \\
100 \mathrm{ccm} \\
\end{array}$} & & & & \\
\hline & & & & & & & & & \multicolumn{3}{|c|}{$\mathrm{g}$ in $100 \mathrm{cem}$} \\
\hline 1 & $\begin{array}{l}\text { Asmanns- } \\
\text { hausen }\end{array}$ & - & Sehiefer & $\begin{array}{c}\text { Spät- } \\
\text { burgunder }{ }^{6} \text { ) }\end{array}$ & - & 127,1 & 1,83 & 1,0124 & 11,32 & 7,890 & $0,54 t$ \\
\hline 2 & Eibingen & Flecht ${ }^{7}$ ) & Tonschiefer & Riesling & 4, Okt. & 93,6 & 1,37 & 0,9992 & 8,98 & 3,630 & 0,211 \\
\hline 3 & " & $78)$ & $"$ & , & 2. Okt. & 76,5 & 1,31 & 1,0006 & 7,53 & 3,450 & 0,21 \\
\hline 4 & $n$ & Leideck & $\mathrm{L} \Theta \mathrm{hm}$ & $\begin{array}{l}\text { Sylvaner } \\
\text { veredelt }\end{array}$ & 29. Sept. & 76,2 & 1,14 & 1,0001 & 7,42 & 2,749 & 0,19 \\
\hline 5 & $"$ & $"$ & $"$ & $\begin{array}{l}\text { Riesling } \\
\text { veredelt }\end{array}$ & 12. okt. & 63,1 & 1,37 & 1,0020 & 6,49 & 3,363 & 0,19 \\
\hline 6 & " & $"$ & $"$ & Sylvaner & 29. Sept. & 60,4 & 0,84 & 1,0001 & 5,94 & 2,577 & $0,22]$ \\
\hline 7 & Geisenheim & Fuchsberg & $"$ & Elbling & 20. Sept. & 68,3 & 1,13 & 1,0002 & 6,48 & 2,828 & 0,22 \\
\hline 8 & Ruidesheim & Burgweg & - & Riesling & - & 105,2 & 1,05 & 0,9989 & 11,27 & 4,420 & $0,26 €$ \\
\hline 9 & , & $\begin{array}{l}\text { Pares und } \\
\text { Zollhaus }\end{array}$ & - & $"$ & - & 125,0 & 1,03 & 0,9985 & 11,92 & 4,688 & $0,31 i$ \\
\hline 10 & Winkel & Gutenberg & Fetter Lehm & $"$ & $\begin{array}{l}\text { 17. n. } 18 \text {. } \\
\text { okt. }\end{array}$ & 85,0 & 1,11 & 0,9977 & 7,66 & 2,650 & $0,18 \varepsilon$ \\
\hline
\end{tabular}

B. Rheintal unterhal

\begin{tabular}{c|c|c|c}
11 & Boppard & $\begin{array}{c}\text { Bopparder } \\
\text { Hamm } \\
\text { (Fesserlay) }\end{array}$ & Tonschiefer \\
12 & Oberwesel & Harthelle \\
13 & $"$ & Rheinhelle & Schiefex \\
& $"$ &
\end{tabular}

\begin{tabular}{c|r} 
Riesling & $16.0 \mathrm{kt}$. \\
$"$ & $8.0 \mathrm{kt}$. \\
& $15.0 \mathrm{kt}$.
\end{tabular}

\begin{tabular}{|c|c|c|}
75,7 & 1,00 & 0,9978 \\
60,9 & 1,10 & 0,9984 \\
64,4 & 1,18 & 0,9983
\end{tabular}

\begin{tabular}{l|l|l|l|}
7,45 & 2,612 & 0,181
\end{tabular}

$6,40 \cdot 1,970 \quad 0,15($ \begin{tabular}{l|l|l}
6,70 & 2,380 & $0,16 t$
\end{tabular}

C. Nabe

\begin{tabular}{|c|c|c|c|c|c|c|c|c|c|c|c|}
\hline 14 & Bretzenheim & Boländer & Fetter Lehm & Gemiseht & 18. Okt. & 75,8 & 0,98 & 0,9980 & 7,64 & 2,279 & $0,16^{r}$ \\
\hline 15 & $"$ & Kronenberg & $"$ & mit Rïesling & 18. Okt. & 69,4 & 0,96 & 0,9983 & 7,43 & 2,983 & $0,21]$ \\
\hline & $"$ & Manik & Lehm & $\begin{array}{l}\text { Gemischt } \\
\text { mit Riesling }\end{array}$ & 18. Okt. & 76,5 & 1,00 & 0,9980 & 7,37 & 2,641 & $0,20 r$ \\
\hline & Monzingen & $\begin{array}{l}\text { Fels und } \\
\text { Lay }\end{array}$ & $\begin{array}{l}\text { Verwitt. } \\
\text { Fels }\end{array}$ & $\begin{array}{c}\text { u. Sylvaner } \\
\text { Riesling }\end{array}$ & 14.-10. Okt. & - & 1,25 & 0,9949 & 9,05 & 2,633 & \\
\hline & Kreuznaeh & Brïekes & $\begin{array}{l}\text { Roter Sand- } \\
\text { stein mit }\end{array}$ & $"$ & 25. Okt. & 86,2 & 0,76 & 0,9939 & 8,82 & 2,389 & \\
\hline & $"$ & Kronenberg & $\begin{array}{l}\text { Leiehter } \\
\text { Letten }\end{array}$ & $"$ & 24. $0 \mathrm{kt}$. & 88,5 & 0,81 & 0,9941 & 8,69 & 2,316 & $0,21^{\mathfrak{z}}$ \\
\hline & Roxheim & Berg & $\begin{array}{l}\text { Sehwerer } \\
\text { Letten }\end{array}$ & $\%$ & 27. Okt. & 92,1 & 0,79 & 0,9936 & 9,30 & 1,699 & 35 \\
\hline
\end{tabular}

D. Moseltal unı \begin{tabular}{l|c|c|c|c|c|c|c|c|c|c|c}
21 & $\begin{array}{c}\text { Canzen } \\
\text { (Saar) }\end{array}$ & Berg & Sehiefer & Riesling & 19. Okt. & 77,6 & 1,29 & 1,0019 & 7,44 & 3,527 & 0,190 \\
22 & Winterich & Gut Geiers- & $\#$ & $*$ & 16. okt. & 76,2 & 0,88 & 0,9972 & $\mathbf{7 , 5 3}$ & $\mathbf{1 , 9 1 6}$ & 0,136
\end{tabular}

1) Extrakt einsehließlich des gesamten vorhandenen Zuckers. ${ }^{2}$ ) Extrakt nach Abzug dr 4) Nichtflüchtige Säure nach Abzug der freien Weinsäure und der Hälfte der halbgebundenen Wei
6) Weiß gekeltert, sogenannter Rotweiß.
7) Starke Edelfärle.
8) Junger Weinberg, mitte 


\section{veine.}

a. 1.

\begin{tabular}{|c|c|c|c|c|c|c|c|c|c|c|c|c|c|c|c|c|}
\hline \multirow{2}{*}{$\begin{array}{c}\text { Ge- } \\
\text { samt- } \\
\text { 3âure, } \\
\text { als } \\
\text { Vein- } \\
\text { sänre } \\
\text { be- } \\
\text { rech- } \\
\text { net }\end{array}$} & \multirow{2}{*}{$\begin{array}{c}\text { Flüch- } \\
\text { tige } \\
\text { Säure, } \\
\text { als } \\
\text { Essig- } \\
\text { sulure } \\
\text { be- } \\
\text { reeh- } \\
\text { net }\end{array}$} & \multirow{2}{*}{$\begin{array}{l}\text { Nicht- } \\
\text { flüh- } \\
\text { tige } \\
\text { särure, } \\
\text { als } \\
\text { Wein- } \\
\text { surare } \\
\text { be- } \\
\text { reuh- } \\
\text { net }\end{array}$} & \multirow{2}{*}{$\begin{array}{l}\text { Ge* } \\
\text { samt- } \\
\text { Wein- } \\
\text { seare }\end{array}$} & \multirow{2}{*}{$\begin{array}{c}\text { Freie } \\
\text { Wein- } \\
\text { sture }\end{array}$} & \multirow{2}{*}{$\begin{array}{l}\text { Wein- } \\
\text { stein }\end{array}$} & \multirow{2}{*}{$\begin{array}{c}\text { An } \\
\text { alka- } \\
\text { lisehe } \\
\text { Erden } \\
\text { gebun- } \\
\text { dene } \\
\text { Wein- } \\
\text { säure }\end{array}$} & \multirow[b]{2}{*}{$\begin{array}{l}\text { Gly- } \\
\text { cerin. }\end{array}$} & \multirow{2}{*}{$\begin{array}{c}\operatorname{In-} \\
\text { vert- } \\
\text { zhuter }\end{array}$} & \multirow{2}{*}{$\begin{array}{l}\text { Schwe- } \\
\text { fel- } \\
\text { säure } \\
\left(\mathrm{SO}_{3}\right)\end{array}$} & \multirow{2}{*}{$\mid$\begin{tabular}{c|} 
Ex- \\
trakt \\
in \\
Sinne \\
des \\
Wein- \\
geset- \\
zes $\left.{ }^{2}\right)$
\end{tabular}} & \multirow{2}{*}{\begin{tabular}{|} 
Ex- \\
trakt ${ }^{3}$ ) \\
nach \\
Abzug \\
der \\
Ge- \\
samb- \\
säure
\end{tabular}} & \multirow{2}{*}{$\begin{array}{c}\text { Ex- } \\
\text { trakt } \\
\text { nach } \\
\text { Abzug } \\
\text { der } \\
\text { nicht- } \\
\text { fluch- } \\
\text { tigen } \\
\text { Sau- } \\
\text { ren }\end{array}$} & \multirow{2}{*}{$\begin{array}{l}\text { Säure- } \\
\text { rest } \\
\text { nach } \\
\text { Mös- } \\
\text { lin- } \\
\text { ger }\end{array}$} & \multicolumn{2}{|c|}{$\begin{array}{c}\text { Verhältnis } \\
\text { von }\end{array}$} & \multirow{3}{*}{$\begin{array}{l}\text { Ab- } \\
\text { nahme } \\
\text { der } \\
\text { Säure } \\
\text { des } \\
\text { Mo- } \\
\text { stes } 5 \\
\\
\%\end{array}$} \\
\hline & & & & & & & & & & & & & & \multirow[t]{2}{*}{$\begin{array}{c}\text { Ex- } \\
\left.\text { trakt }^{3}\right) \\
\text { :Asche } \\
=100\end{array}$} & \multirow[t]{2}{*}{$\begin{array}{l}\text { Alko- } \\
\text { hol : } \\
\text { Glyee- } \\
\text { rim = } \\
100:\end{array}$} & \\
\hline \multicolumn{14}{|c|}{$\mathrm{sin} 100 \mathrm{~cm}$} & & & \\
\hline 1,67 & 0,123 & 1,516 & 0,056 & 0 & 0,070 & 0 & 1,670 & 2,632 & 0,020 & 5,358 & 8,688 & 3,842 & 1,488 & 10,2 & 14,8 & 17,2 \\
\hline 1,18 & 0,042 & 1,127 & 0,506 & 0,856 & 0,193 & 0 & 1,152 & 0,288 & 0,024 & 3,442 & 2,262 & 2,315 & 0,696 & 6,1 & 12,8 & 17,8 \\
\hline 1,18 & 0,072 & 1,090 & 0,323 & 0,135 & 0,028 & 0,165 & 0,886 & 0,240 & 0,024 & 3,810 & 2,130 & 2,220 & 0,861 & 6,5 & 11,7 & 16,8 \\
\hline 1,05 & 0,085 & 0,944 & 0,278 & 0,068 & 0,113 & 0,120 & 0,581 & 0,111 & 0,012 & 2,738 & 1,688 & 1,794 & 0,771 & 6,9 & 7,8 & 17,2 \\
\hline 1,15 & 0,069 & 1,064 & 0,428 & 0,165 & 0,141 & 0,150 & 0,881 & 0,232 & 0,012 & 3,231 & 2,081 & 2,167 & 0,767 & 5,9 & 13,6 & 22,3 \\
\hline 0,84 & 0,099 & 0,716 & 0,248 & 0,068 & 0,113 & 0,090 & 0,551 & 0,149 & 0,021 & 2,528 & 1,688 & 1,812 & 0,558 & 8,7 & 9,3 & 14,8 \\
\hline 0,81 & 0,070 & 0,722 & 0,233 & 0 & 0,150 & 0,113 & 0,581 & 0,178 & 0,010 & 2,750 & 1,940 & 2,098 & 0,605 & 8,2 & 9,0 & 36,1 \\
\hline 0,95 & 0,094 & 0,832 & 0,068 & 0 & 0,085 & 0 & 1,251 & 0,402 & 0,028 & 4,118 & 3,168 & 3,286 & 0,798 & 6,5 & 11.1 & 20,8 \\
\hline 0,93 & 0,120 & 0,780 & 0,056 & 0 & 0,070 & 0 & 1,448 & 0,511 & 0,031 & 4,227 & 3,297 & 3,447 & 0,752 & 7,4 & 12,1 & 24,3 \\
\hline 0,91 & 0,090 & 0,797 & 0,409 & 0,221 & 0,061 & 0,139 & 0,719 & 0,044 & 0,017 & 2,650 & 1,740 & 1,853 & 0,427 & 7,0 & 9,4 & 28,2 \\
\hline
\end{tabular}

les Rheingaues.

\begin{tabular}{|c|c|c|c|c|c|c|c|c|c|c|c|c|c|c|c|c|}
\hline 0,93 & 0,053 & 0,864 & 0,319 & 0,131 & 0,103 & $\mid 0,101$ & 0,708 & $|0,177|$ & 0,007 & 2,585 & $\mid 1,585$ & 1,601 & 0,639 & 7,1 & 9,5 & 13,6 \\
\hline & & 0,810 & & 0,283 & 0,103 & 0,068 & 0,773 & 0,084 & 0,015 & 1,970 & 1,050 & 1,160 & 0,502 & 7,6 & 12,1 & \\
\hline 94 & 0,072 & 0,850 & 0,266 & 0,086 & 0,103 & 0,098 & 0,637 & 0,146 & 0,019 & 2,334 & 1,394 & 1,484 & 0,674 & 7,1 & 9,5 & 28,0 \\
\hline
\end{tabular}

a I.

\begin{tabular}{l|l|l|l|l|l|l|l|l|l|l|l|l|l|l|l|l|l|}
0,74 & 0,127 & 0,581 & 0,154 & 0 & 0,141 & 0,041 & 0,857 & 0,190 & 0,009 & 2,189 & 1,449 & 1,508 & 0,504 & 7,6 & 11,2 & 41,7 \\
0,78 & 0,115 & 0,636 & 0,158 & 0 & 0,132 & 0,053 & 0,852 & 0,217 & 0,010 & 2,866 & 2,086 & 2,230 & 0,557 & 7,4 & 11,5 & 33,8 \\
0,68 & 0,072 & 0,590 & 0,188 & 0 & 0,122 & 0,090 & 0,932 & 0,149 & 0,020 & 2,592 & 1,912 & 2,002 & 0,496 & 8,0 & 12,6 & 41,0 \\
0,85 & 0,052 & 0,785 & 0,248 & 0,098 & 0,081 & 0,083 & 0,879 & 0,190 & - & 2,543 & 1,693 & 1,758 & 0,612 & 5,3 & 9,7 & 37,2 \\
0,57 & 0,076 & 0,475 & 0,143 & 0 & 0,122 & 0,045 & 0,928 & 0,183 & 0,036 & 2,356 & 1,786 & 1,881 & 0,408 & 9,3 & 10,5 & 37,5 \\
0,62 & 0,084 & 0,515 & 0,158 & 0 & 0,103 & 0,075 & 0,786 & 0,103 & 0,028 & 2,313 & 1,698 & 1,798 & 0,436 & 9,3 & 9,0 & 36,4 \\
0,55 & 0,074 & 0,457 & 0,120 & 0 & 0,103 & 0,038 & 0,899 & 0,188 & 0,029 & 1,611 & 1,061 & 1,154 & 0,397 & 8,6 & 9,7 & 42,2
\end{tabular}

Nebenflüsse.

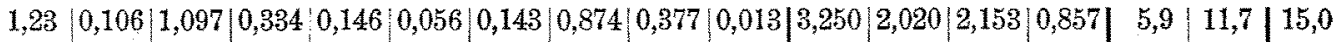
\begin{tabular}{l|l|l|l|l|l|l|l|l|l|l|l|l|l|l|l|l|l|l|l|l|l|l|l|l|l|l|}
0,77 & 0,059 & 0,696 & 0,203 & 0,038 & 0,155 & 0,041 & 0,760 & 0,196 & 0,013 & 1,820 & 1,050 & 1,124 & 0,575 & 7,5 & 10,1 & 20,5
\end{tabular}

$1,1 \mathrm{~g}$ in $100 \mathrm{cem}$ Wein übersteigenden Zuckers.

3) Hixtrakt im Sinne des Weingesetzes. äure. 5) Dieser Berechnung ist die nichtfluchtige Säure des Weines zugrunde gelegt.

tarke Fäulnis: 


\begin{tabular}{|c|c|c|c|c|c|c|c|c|c|c|c|}
\hline \multirow{3}{*}{ No. } & \multirow{3}{*}{$\begin{array}{c}\text { Ge- } \\
\text { markung }\end{array}$} & \multirow{3}{*}{$\begin{array}{c}\text { Lage } \\
\text { (Gewann) }\end{array}$} & \multirow{3}{*}{ Bodenart } & \multirow{3}{*}{$\begin{array}{l}\text { Trauben- } \\
\text { sorte }\end{array}$} & \multirow{3}{*}{$\begin{array}{c}\text { Zeit } \\
\text { der Lese }\end{array}$} & \multicolumn{2}{|c|}{$\begin{array}{l}\text { Zusammen- } \\
\text { setzung des } \\
\text { Mostes }\end{array}$} & \multirow{3}{*}{$\begin{array}{c}\text { Spez. } \\
\text { Ge- } \\
\text { wicht } \\
\text { bei } \\
15^{0} \mathrm{C} . \\
\end{array}$} & & \multirow{2}{*}{$\begin{array}{c}\text { Ge- } \\
\text { samt- } \\
\text { Ex- } \\
\text { trakt }\end{array}$} & \multirow{2}{*}{$\begin{array}{l}\text { Sline- } \\
\text { ralbe- } \\
\text { stand- } \\
\text { teile }\end{array}$} \\
\hline & & & & & & \multirow{2}{*}{$\begin{array}{c}\text { Most- } \\
\text { Ge- } \\
\text { wieht } \\
\\
\text { (Grade } \\
\text { Örehsle) } \\
\end{array}$} & \multirow{2}{*}{$\begin{array}{c}\text { Säure } \\
\text { (als } \\
\text { wein- } \\
\text { säure } \\
\text { berech- } \\
\text { net) } \\
\text { g in } \\
100 \mathrm{ccm} \\
\end{array}$} & & $\begin{array}{c}\text { Al- } \\
\text { kohol }\end{array}$ & & \\
\hline & & & & & & & & & \multicolumn{3}{|c|}{$\mathrm{g}$ in $100 \mathrm{ccm}$} \\
\hline 23 & $\begin{array}{c}\text { Wittlich } \\
\text { (Liesertal) }\end{array}$ & Knuth & Schiefer & $\begin{array}{l}\text { Riesling } \\
\text { und Elbling }\end{array}$ & 11. $-23.0 \mathrm{kt}$ & 68,6 & 1,12 & 0,9985 & 6,58 & 2,360 & 0,191 \\
\hline 24 & $"$ & $\begin{array}{c}\text { Portners- } \\
\text { berg }\end{array}$ & $"$ & $\begin{array}{c}\text { Riesling, } \\
\text { Elbling umd } \\
\text { Sylvaner }\end{array}$ & $"$ & 71,6 & 1,18 & 0,9976 & 6,47 & 2,081 & $0,16 \%$ \\
\hline 25 & $"$ & $"$ & $"$ & $\begin{array}{c}\text { Riesling } \\
\text { und } \\
\text { Burgunder }\end{array}$ & $"$ & 65,0 & 1,14 & 0,9978 & 6,74 & 2,345 & $0,17 \varepsilon$ \\
\hline & Zeltingen & Burg & $"$ & Riesling & 25. Okt. & 73,2 & 0,87 & 0,9982 & 7,03 & 2,700 & 0,201 \\
\hline
\end{tabular}

E. Ostdeutsche

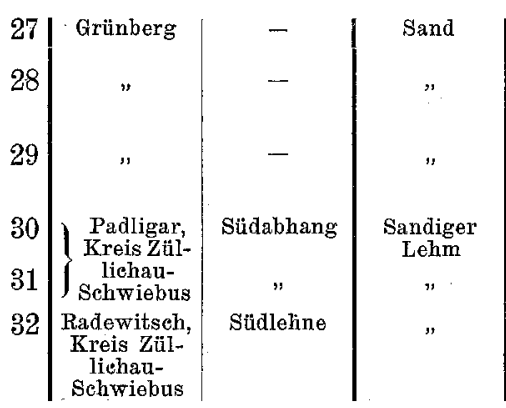

\begin{tabular}{c|c|c|c|c|c}
80,6 & 0,65 & 0,9925 & 9,04 & 2,096 & 0,174 \\
92,4 & 0,85 & 0,9938 & 8,96 & 2,472 & 0,147 \\
83,6 & 0,56 & 0,9934 & 8,90 & 2,230 & $0,22 \varepsilon$ \\
& & & & & \\
74,1 & 0,64 & 0,9940 & 7,83 & 1,760 & $0,18 €$ \\
87,9 & 0,64 & 0,9927 & 9,27 & 2,210 & $0,21 \varepsilon$ \\
80,0 & 0,67 & 0,9921 & 9,02 & 1,948 & $0,16 \mathrm{C}$ \\
& & & & &
\end{tabular}

F. Rhein

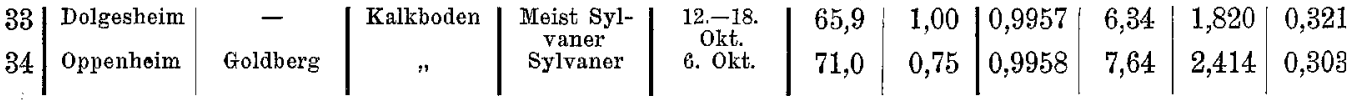

\section{G. Unter}

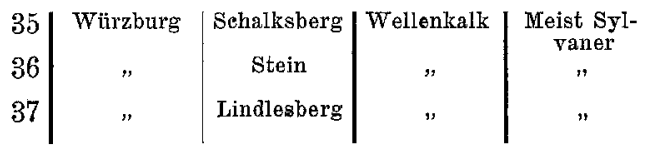

$$
\text { 28. Okt. }
$$

\begin{tabular}{l|l|l|l|l|l}
66,4 & 0,99 & 1,0001 & 6,34 & 2,950 & 0,243 \\
72,5 & 0,88 & 0,9983 & 7,94 & 3,100 & 0,281 \\
71,3 & 0,92 & 0,9982 & 7,36 & 2,769 & 0,236
\end{tabular}

H. Rhein

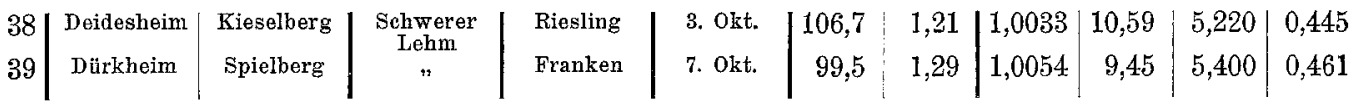

I. Würt.

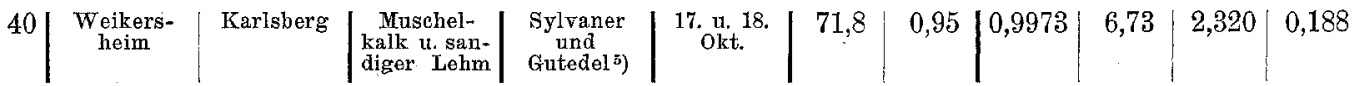




\begin{tabular}{|c|c|c|c|c|c|c|c|c|c|c|c|c|c|c|c|c|}
\hline 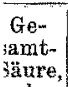 & $\begin{array}{l}\text { Flüch- } \\
\text { tige } \\
\text { Säure, }\end{array}$ & $\begin{array}{l}\text { Nicht- } \\
\text { flach- } \\
\text { tige } \\
\text { sanre }\end{array}$ & & & & An & & & & $\underset{\substack{\mathrm{Ex}-\\
\text { trakt } \\
\text { im }}}{ }$ & $\begin{array}{c}\text { Exw } \\
\text { trakt }\end{array}$ & $\begin{array}{l}\text { Ex- } \\
\text { trakt } \\
\text { nath } \\
\text { than }\end{array}$ & Säure- & \multicolumn{2}{|c|}{$\begin{array}{l}\text { Verhältnis } \\
\text { ₹on }\end{array}$} & \multirow{2}{*}{$\begin{array}{l}\text { Ab- } \\
\text { nahme } \\
\text { der } \\
\text { Säure } \\
\text { des } \\
\text { Mo- } \\
\text { stes }\end{array}$} \\
\hline $\begin{array}{l}\text { Vein- } \\
\text { säure } \\
\text { be- } \\
\text { rech- } \\
\text { net }\end{array}$ & $\begin{array}{l}\text { Essig- } \\
\text { säure } \\
\text { be- } \\
\text { rech- } \\
\text { net }\end{array}$ & $\begin{array}{l}\text { Wein- } \\
\text { süure } \\
\text { be- } \\
\text { rech- } \\
\text { net }\end{array}$ & $\begin{array}{l}\text { Wean- } \\
\text { Wäure }\end{array}$ & $\begin{array}{l}\text { Wein- } \\
\text { süure }\end{array}$ & stein & $\begin{array}{l}\text { gebun- } \\
\text { dene } \\
\text { Wein- } \\
\text { säure }\end{array}$ & cerin & \begin{tabular}{|} 
vert- \\
zucker
\end{tabular} & $\begin{array}{l}\text { säure } \\
\left(\mathrm{SO}_{3}\right)\end{array}$ & $\mid \begin{array}{c}\text { des } \\
\text { Wein- } \\
\text { geset- } \\
\text { zes }\end{array}$ & $\begin{array}{l}\text { der } \\
\text { Ge- } \\
\text { samt- } \\
\text { samare }\end{array}$ & $\begin{array}{l}\text { nicht- } \\
\text { fiüch- } \\
\text { tigen } \\
\text { Säu- } \\
\text { ren } \\
\end{array}$ & $\begin{array}{l}\text { Mös- } \\
\text { lin- } \\
\text { ger }\end{array}$ & \multirow{2}{*}{\multicolumn{2}{|c|}{$\begin{array}{cc}\text { Ex- } & \text { Alko- } \\
\text { trakt } & \text { hol: } \\
\text { : Asehe } & \text { Glyee- } \\
=100: & \text { rin }= \\
= & 100:\end{array}$}} & \\
\hline \multicolumn{14}{|c|}{$\mathrm{g}$ in $100 \mathrm{cem}$} & & & $\%$ \\
\hline 1,00 & 0,047 & 0,941 & 0,304 & 0,049 & 0,132 & 0,150 & 0,605 & 0,094 & - & 2,360 & 1,360 & 1,419 & 0,764 & 8,1 & 9,2 & 16,0 \\
\hline 0,97 & 0,050 & 0,907 & 0,360 & 0,113 & 0,122 & 0,150 & 0,581 & 0,062 & 一 & 2,081 & 1,111 & 1,174 & 0,670 & 7,8 & 9,0 & 23,1 \\
\hline 0,99 & 0,040 & 0,940 & 0,326 & 0,101 & 0,122 & 0,128 & 0,595 & 0,086 & - & 2,345 & 1,355 & 1,405 & 0,726 & 7,3 & 8,8 & 17,5 \\
\hline 0,65 & 0,074 & 0,557 & 0,176 & 0 & 0,127 & 0,075 & 0,860 & 0,168 & 0,017 & 2,632 & 1,982 & 2,075 & 0,469 & 7,6 & 12,2 & 24,5 \\
\hline
\end{tabular}

Vein baugebiet.

\begin{tabular}{|c|c|c|c|c|c|c|c|c|c|c|c|c|c|c|c|}
\hline $0,58: 0,080$ & 0,430 & $|0,214|$ & 0 & 0,132 & 0,105 & 0,939 & 0,086 & - & 2,096 & 1,566 & 1,666 & $|0,323|$ & 8,3 & 10,4 & 33,8 \\
\hline 0,112 & 0,550 & 0,296 & 0,169 & 0,038 & 0,098 & 1,297 & 0,096 & - & 2,472 & 1,802 & 1,942 & 0,297 & 5,9 & 14,5 & 37,6 \\
\hline 0,044 & 0,505 & 0,229 & 0,064 & 0,160 & 0,038 & 0,741 & $\begin{array}{l}\text { Spu- } \\
\text { ren }\end{array}$ & 0,026 & 2,230 & 1,670 & 1,725 & $0,858\}$ & 10,0 & 8,3 & 9,8 \\
\hline 0,120 & 0,600 & 0,286 & 0,086 & 0,141 & 0,038 & 0,638 & 0,117 & 0,016 & 1,748 & 1,048 & 1,143 & 0,439 & 10,7 & 8,1 & 6,2 \\
\hline 0,061 & 0,584 & 0,169 & 0,004 & 0,085 & 0,098 & 0,791 & 0,107 & 0,024 & 2,208 & 1,543 & 1,619 & 0,497 & 9,6 & 8,5 & 8,1 \\
\hline 0,077 & 0,614 & 0,244 & 0,045 & 0,122 & 0,101 & 0,580 & 0,064 & 0,007 & 1,948 & 1,238 & 1,334 & 0,469 & 8,2 & 6,4 & 8,4 \\
\hline
\end{tabular}

essen.

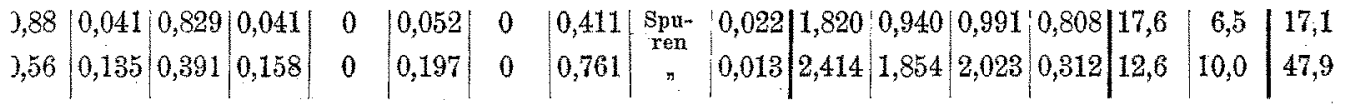

ranken.

\begin{tabular}{l|l|l|l|l|l|l|l|l|l|l|l|l|l|l|l|l|l|l|l|l|l|l|}
3,69 & 0,104 & 0,560 & 0,131 & 0 & 0,166 & 0 & 0,896 & 0,126 & 0,011 & 2,924 & 2,234 & 2,364 & 0,494 & 8,3 & 14,1 & 43,4
\end{tabular} \begin{tabular}{l|l|l|l|l|l|l|l|l|l|l|l|l|l|l|l|l|l|l|l|l|l|}
3,64 & 0,084 & 0,585 & 0,088 & 0 & 0,110 & 0 & 0,732 & 0,135 & 0,018 & 3,065 & 2,425 & 2,580 & 0,491 & 9,2 & 9,2 & 39,2
\end{tabular} \begin{tabular}{l|l|l|l|l|l|l|l|l|l|l|l|l|l|l|l|l|l|l|l|l|}
3,80 & 0,105 & 0,669 & 0,208 & 0 & 0,165 & 0,077 & 0,870 & 0,049 & 0,018 & 2,769 & 1,969 & 2,100 & 0,565 & 8,5 & 11,8 & 27,8
\end{tabular}

falz.

\begin{tabular}{l|l|l|l|l|l|l|l|l|l|l|l|l|l|l|l|l|l|l|l|l|l|l|l|l|l|l|l|l|}
1,19 & 0,086 & 1,082 & 0,090 & 0 & 0,113 & 0 & 1,222 & 0,320 & 0,087 & 5,000 & 3,810 & 3,918 & 1,037 & 8,9 & 11,7 & 10,6
\end{tabular} \begin{tabular}{l|l|l|l|l|l|l|l|l|l|l|l|l|l|l|l|l|l|l|l|l|l|l|}
1,15 & 0,063 & 1,071 & 0,090 & 0 & 0,113 & 0 & 1,249 & 0,255 & 0,024 & 5,245 & 4,095 & 4,174 & 1,026 & 8,8 & 13,2 & 17,0
\end{tabular}

z $m$ berg.

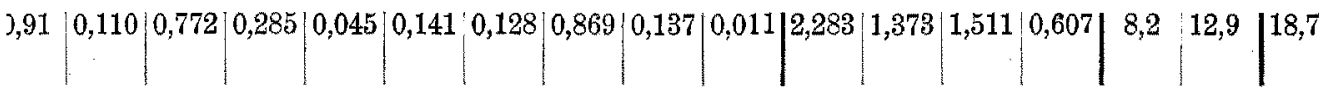

artrauben und Traminer. *) Elbling, Riesling und Traminer. ${ }^{5}$ ) Nur gesunde Trauben. 


\begin{tabular}{|c|c|c|c|c|c|c|c|c|c|c|c|}
\hline \multirow{3}{*}{ No. } & \multirow{3}{*}{$\begin{array}{c}\text { Ge- } \\
\text { markung }\end{array}$} & \multirow{3}{*}{$\begin{array}{c}\text { Lage } \\
\text { (Gewann) }\end{array}$} & \multirow{3}{*}{ Bodenart } & \multirow{3}{*}{$\begin{array}{l}\text { Trabuben- } \\
\text { sorte }\end{array}$} & \multirow{3}{*}{$\begin{array}{c}\text { Zeit } \\
\text { der Lese }\end{array}$} & \multicolumn{2}{|c|}{$\begin{array}{l}\text { Zusammen- } \\
\text { setzung des } \\
\text { Mostes }\end{array}$} & \multirow{3}{*}{$\begin{array}{c}\text { Spez. } \\
\text { Ge- } \\
\text { wieht } \\
\text { bei } \\
15^{\circ} \mathrm{O} .\end{array}$} & & \multirow[b]{2}{*}{$\begin{array}{l}\text { Ge- } \\
\text { samt- } \\
\text { Ex- } \\
\text { trakt }\end{array}$} & \multirow{2}{*}{$\begin{array}{l}\text { Mine- } \\
\text { ralbe- } \\
\text { stand- } \\
\text { teile }\end{array}$} \\
\hline & & & & & & \multirow{2}{*}{$\begin{array}{c}\text { Most- } \\
\text { Ge- } \\
\text { wicht } \\
\text { (Grade } \\
\text { Öchsle) }\end{array}$} & \multirow{2}{*}{$\begin{array}{c}\text { Sänire } \\
\text { (als } \\
\text { Wein- } \\
\text { säure } \\
\text { berech- } \\
\text { net) } \\
\text { g in } \\
100 \mathrm{cem}\end{array}$} & & $\begin{array}{c}\text { Al- } \\
\text { kohol }\end{array}$ & & \\
\hline & & & & & & & & & \multicolumn{3}{|c|}{$g$ in $100 \mathrm{ecm}$} \\
\hline 41 & $\begin{array}{l}\text { Weikers- } \\
\text { heim }\end{array}$ & Karlsberg & $\begin{array}{l}\text { Musehel- } \\
\text { kalk u. san- } \\
\text { diger Lehm }\end{array}$ & $\begin{array}{l}\text { Sylvaner } \\
\text { und } \\
\text { Gutedel }{ }^{1} \text { ) }\end{array}$ & $\begin{array}{l}\text { 17. u. } 18 . \\
\text { okt. }\end{array}$ & 74,7 & 1,16 & 0,9999 & 7,12 & 2,990 & 0,226 \\
\hline 42 & $"$ & $"$ & $"$ & Süfroth 2 ) & 10. 0kt. & 78,6 & 1,11 & 0,9993 & 7,87 & 3,040 & 0,15 \\
\hline 43 & $"$ & Schmecker & $\begin{array}{l}\text { Schwerer } \\
\text { Lehm }\end{array}$ & $\begin{array}{l}\text { Sylvaner } \\
\text { und } \\
\text { Gutedel }{ }^{3} \text { ) }\end{array}$ & 22. Okt. & 72,9 & 0,90 & 0,9962 & 7,19 & 2,140 & $0,18 \mathrm{t}$ \\
\hline
\end{tabular}

K. $E l$ a $\mathbb{B}$

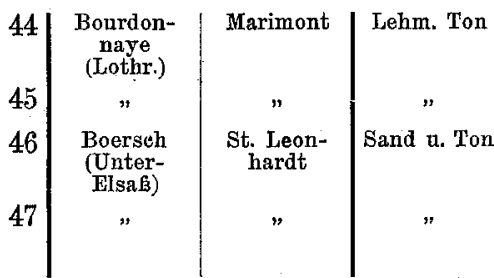

\begin{tabular}{l|c|c|c}
44 & $\begin{array}{c}\text { Bourdon- } \\
\text { naye } \\
\text { (Lothr.) }\end{array}$ & Marimont & Lehm. Ton \\
45 & $"$ & $"$ & $"$ \\
46 & $\begin{array}{c}\text { Boerseh } \\
\text { (Unter- } \\
\text { Elsak) }\end{array}$ & $\begin{array}{c}\text { St. Leon- } \\
\text { hardt }\end{array}$ & Sand u. Ton \\
& $"$ & $n$ &
\end{tabular}

64,4
75,5
67,8
58,8

\begin{tabular}{c|c|c}
6,53 & 2,370 & $0,23 \varepsilon$ \\
7,57 & 3,870 & 0,44 \\
7,15 & 1,754 & 0,175 \\
6,32 & 1,504 & $0,16 c$
\end{tabular}

L. Sonstig $48\left|\begin{array}{c|c}\text { Erbstadt, } \\ \text { Kreis } \\ \text { Hanau }\end{array}\right| \begin{gathered}\text { Schlof } \\ \text { Naumburg }\end{gathered} \mid \begin{gathered}\text { Roter } \\ \text { Tonsehiefer }\end{gathered}$
Riesling $\mid$\begin{tabular}{ll|} 
Ende Okt. & 80,6
\end{tabular}

$0,\left.95\right|^{0,9962}$

\section{Rot}

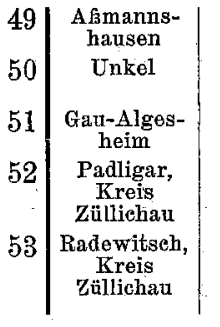

Basalt
Kalk und
Letten
Sand und
Lehm
$"$

\begin{tabular}{|c|c|c|}
\hline patbur- & - & 77,6 \\
\hline " & $\begin{array}{l}\text { 25. Sept. bis } \\
\text { 6. Okt. }\end{array}$ & - \\
\hline $\begin{array}{l}\text { Frühbur- } \\
\text { gunder }\end{array}$ & - & 87,0 \\
\hline $\begin{array}{l}\text { Blauschön- } \\
\text { edel und } \\
\text { Kuržrot }\end{array}$ & 17. Okt. & 87,6 \\
\hline $\begin{array}{l}\text { Burgunder } \\
\text { und } \\
\text { Kurzrot }\end{array}$ & 7. $-10.0 \mathrm{kt}$. & 89,0 \\
\hline
\end{tabular}

2) Weiß gekeltert (Klaret).

\begin{tabular}{c|c|c|}
0,95 & 0,9984 & 7,97 \\
- & 0,9970 & 8,67 \\
0,92 & 0,9928 & 9,02 \\
0,70 & 0,9948 & 8,91 \\
0,75 & 0,9966 & 9,27
\end{tabular}

\begin{tabular}{l|l|l}
2,946 & 0,319
\end{tabular}

$2,946 \quad 0,222$

2,484

2,380

2,990 0,296 0,211

3) Nur gesunde Trauben.

[Fortsetzung von S. 297.]

Grenzzahl für den Extraktgehalt nach Abzug der nichtflüchtigen Säuren $(1,1 \mathrm{~g}$ in $100 \mathrm{ccm})$. Sie wurde unterschritten bei drei Weinen: No. 33 mit $0,991 \mathrm{~g}$, No. 47 mit $0,883 \mathrm{~g}$ und No. 48 mit 0,900 g Extrakt nach Abzug der nichtflüchtigen Säuren. Sechs Weine kamen der Grenzzahl nahe: No. 12 mit 1,160 g, No. 20 mit $1,154 \mathrm{~g}$, No, 22 mit 1,124 g, No. 24 mit 1,174 g, No. 30 mit 1,143 g, No. 46 mit 1,110 g Extrakt nach Abzug der nichtflüchtigen Säuren in $100 \mathrm{ccm}$. Es sind dies mit Ausnabme von No. 24 dieselben Weine, bei denen auch der Extrakt nach Abzug der Gesamtsäure der Grenzzahl nahe war. Da diese Weine sämtlich Jung- 


\begin{tabular}{|c|c|c|c|c|c|c|c|c|c|c|c|c|c|c|c|c|}
\hline $\begin{array}{l}\text { ye- } \\
\text { mt- } \\
\text { iure, } \\
\text { als } \\
\text { ein- } \\
\text { iure } \\
\text { oe- } \\
\text { en- } \\
\text { let }\end{array}$ & $\begin{array}{c}\text { Flüch- } \\
\text { tige } \\
\text { Sä̈ure, } \\
\text { als } \\
\text { Essig- } \\
\text { säure } \\
\text { be- } \\
\text { reeh- } \\
\text { net }\end{array}$ & \begin{tabular}{|c|} 
Nicht- \\
flüeh- \\
tige \\
Säure, \\
als \\
Wein- \\
süure \\
be- \\
rech- \\
net
\end{tabular} & \begin{tabular}{|c|} 
\\
Ge- \\
samb- \\
Wein- \\
săture \\
\end{tabular} & $\begin{array}{l}\text { Freie } \\
\text { Wom- } \\
\text { säure }\end{array}$ & $\begin{array}{l}\text { Wein- } \\
\text { stein }\end{array}$ & $\begin{array}{c}\text { An } \\
\text { alka- } \\
\text { lische } \\
\text { Erden } \\
\text { gebun- } \\
\text { dene } \\
\text { Woin- } \\
\text { särure }\end{array}$ & $\begin{array}{l}\text { Qly- } \\
\text { eerin }\end{array}$ & $\begin{array}{c}\text { In- } \\
\text { vert- } \\
\text { zucker }\end{array}$ & $\begin{array}{c}\text { Sehwe- } \\
\text { fel- } \\
\text { säure } \\
\left(\mathrm{SO}_{3}\right)\end{array}$ & $\begin{array}{c}\text { Fx- } \\
\text { trakt } \\
\text { im } \\
\text { Sinne } \\
\text { des } \\
\text { Wein- } \\
\text { geset- } \\
\text { zes }\end{array}$ & $\begin{array}{c}\text { Ex- } \\
\text { trakt } \\
\text { naeh } \\
\text { Abzug } \\
\text { der } \\
\text { Ge- } \\
\text { sant- } \\
\text { sarure }\end{array}$ & $\begin{array}{c}\text { Jx- } \\
\text { takt } \\
\text { nach } \\
\text { Abzug } \\
\text { der } \\
\text { nicht- } \\
\text { flüch- } \\
\text { tigen } \\
\text { Säu- } \\
\text { ren }\end{array}$ & $\begin{array}{c}\text { Säure- } \\
\text { rest } \\
\text { naeh } \\
\text { Mos - } \\
\text { lin- } \\
\text { gon }\end{array}$ & $\begin{array}{l} \\
\text { Exw- } \\
\text { trakt } \\
\text { Asche } \\
=100\end{array}$ & $\begin{array}{l}\text { Alko- } \\
\text { hol : } \\
\text { Giyee- } \\
\text { rin = } \\
100:\end{array}$ & \multirow[t]{2}{*}{$\begin{array}{c}\text { Ab- } \\
\text { nahme } \\
\text { der } \\
\text { Säure } \\
\text { des } \\
\text { Mo- } \\
\text { stos } \\
\\
\text { \% }\end{array}$} \\
\hline \multicolumn{14}{|c|}{$g$ in $100 \mathrm{cem}$} & & & \\
\hline 93 & 0,100 & 0,805 & 0,214 & 0 & 0,177 & 0,072 & 1,089 & 0,165 & 0,013 & 2,925 & 1,995 & 2,120 & 0,698 & 7,6 & 15,3 & 30,6 \\
\hline, 96 & 0,074 & 0,867 & 0,431 & 0,289 & 0,056 & 0,098 & 0,918 & 0,180 & 0,022 & 2,960 & 2,000 & 2,093 & 0,507 & 5,2 & 11,7 & 21,9 \\
\hline .65 & 0,054 & 0,582 & 0,248 & 0,015 & 0,188 & 0,089 & 0,531 & 0,089 & 0,024 & 2,140 & 1,490 & 1,558 & 0,450 & 8,7 & 7,4 & 35,3 \\
\hline
\end{tabular}

othringen.

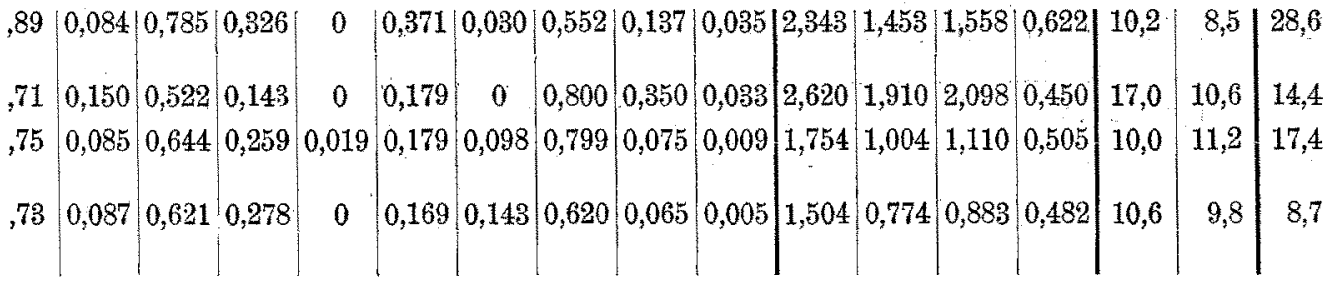

reibweine.

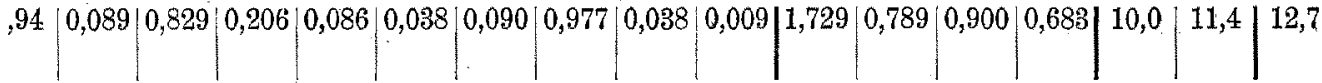

\section{eine.}

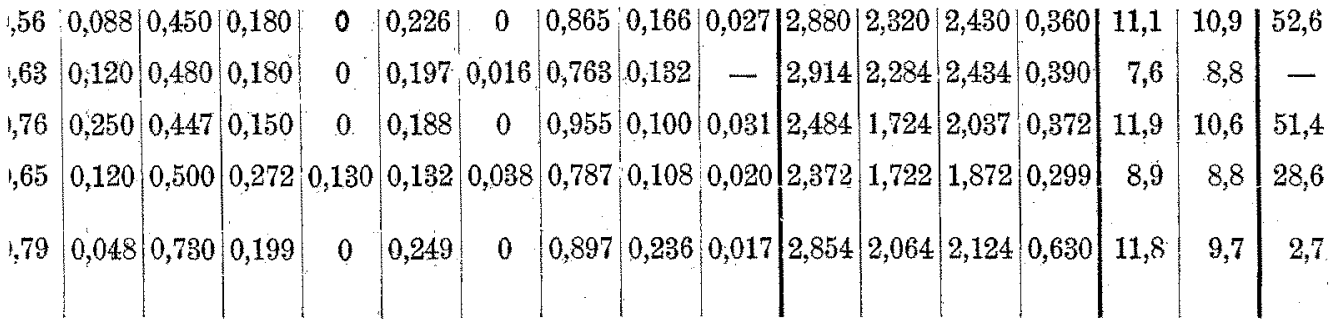

weine nach dem ersten Abstiche sind, ist anzunehmen, dab die Mehrzahl derselben unter dem Einflusse dex Veränderungen, die beim weiteren Lagern in den Weinen vor sich gehen und stets eine weitere Verminderung des Extraktes and der Säure im Gefolge haben; bis zur. Flaschenreife noch unter die gesetzlichen Grenzzahlen heruntergehen werden.

Die neue Grenzzahl für die Mineralbestandteile $(0,13 \mathrm{~g}$ in $100 \mathrm{~cm})$ wurde von allen Weinen erreicht, in drei Fällén jedoch nur wenig überschritten: No. 17 mit $0,136, \mathrm{~g}$, No, 20 mit $0,139, \mathrm{~g}$, No. 22 mit $0,136 \mathrm{~g}$ Mineralbestandteilen in $100 \mathrm{ccm}$ : diese drei Weine erreichen die frühere Grenzzahl $(0,14 \mathrm{~g}$ in $100 \mathrm{~cm})$ nicht. 
Die Rheingauer Weine sind, wie in allen Jahren, reich an Extrakt und Säuren, auch an Glycerin, aber meist nicht sehr reich an Mineralstoffen, wie das Verhältnis von Extrakt zu Mineralstoffen lehrt. Sehr beachtenswert sind die Ergebnisse der Weine des ostdeutschen Weinbaugebietes (No. 27 bis 32). Sie zeichnen sich durch hohen Alkoholgehalt und meist niedrigen Säuregehalt aus; sonst haben diese Weine in der Regel viel Säure und wenig Alkohol. Die Erzeugnisse des Jahres 1901 lehren, dass man auch in dem klimatisch weniger begünstigten ostdeutschen Gebiete nnter günstigen Witterungsverhältnissen einen recht trinkbaren Wein erzielen kann. Die R otwe in e waren, wie stets, reich an Extrakt und Mineralstoffen, aber verhältnismäßig arm an Säure. Nur ein Rotwein enthielt freie Weinsäure, zwei kleine Mengen an alkalische Erden gebundene Weinsäure.

Das Verhältnis von Extrakt zu Mineralstoffen schwankte bei allen Weinen von $100: 5,3$ bis $100: 17,6$; kalkreiche Böden liefern in der Regel mineralstoffreiche Weine. Das Verhältnis von Alkohol zu Glycerin schwankte zwischen $100: 6,4$ und 100:15,3. Man sieht, welchen außerordentlich großen Schwankungen viele Verhältniszahlen unterworfen sind; sie können daher nur mit allergrößter Vorsicht bei der Beurteilung der Handelsweine herangezogen werden.

Ein ganz unnormales Verhalten zeigen die Weine des Jahres 1901 bezüglich ihres Gehaltes an flüchtigen Säuren. Bei Jungweinen nach den ersten Abstichen soll der Gehalt an flüchtigen Säuren noch sehr gering sein; beim Lagern nimmt er in der Regel zu. Von den 53 untersuchten Jungweinen des Jahres 1901 hatten demgegenüber 14 Proben $0,08-0,10 \mathrm{~g}$ und nicht weniger als 17 Proben über $0,10 \mathrm{~g}$, steigend bis zu $0,25 \mathrm{~g}$ flüchtigen Süuren in $100 \mathrm{ccm}$. Einige von den Weinen waren bereits vollständig stichig. Die Ursache für den hohen Essigsäuregehalt der 1901-er Weine ist in den abnormen Witterungsverhältnissen dieses Jahres zu suchen, welche eine starke Fäulnis der Trauben und die frühe Lese bei warmer Witterung zur Folge hatten. Die Fäulnispilze zerstören die Häute der Beeren und lassen das Beerenfleisch zu Tage treten, auf dem sich Hefen und Bakterien aller Art ansiedeln. Infolge der höheren Temperatur tritt in den Trauben schon vor dem Mahlen und noch mehr in der gemahlenen Maische vor dem Keltern Gärung ein, zumal wenn die Trauben bezw. die Maische längere Zeit stehen. Auch die Bakterien beginnen alsbald ihre Tätigkeit, unter ihnen namentlich die Essigbakterien, die den durch die Gärung entstandenen Alkohol in Essigsäure umwandeln. Nach dem Abkeltern des Mostes setzen die Essigbakterien ihre unheilvolle Tätigkeit fort, sofern nicht für gute Abhaltung der Luft gesorgt wird. Bei Rotweinen, die auf den BeerenhüIsen vergären müssen, ist die Gefahr des Essigstiches noch viel gröler. Wir konnten bei mehreren Mosten des Jahres 1901 feststellen, daß dieselben bereits vor dem Abkeltern stichig geworden waren. Die Befürchtung, daß die 1901-er Weine ihren Besitzern noch erhebliche Schwierigkeiten bereiten würden, hat sich im vollen Umfange bestätigt; bereits bei dem ersten Abstiche, der teilweise schon im Dezember vorgenommen wurde, kamen viele kranke Weine zu Tage. Zahlreiche stichige, zähe und rahne (braun gewordene) Weine, sowie solche mit Geruchs- und Geschmacksfehlern wurden der önochemischen Versuchsstation zur Begutachtung eingesandt.

Von fast allen Weinen war auch der zugehörige Most untersucht worden, so daß es möglich ist, die Säureabnahme während der Gärung und beim Lagern bis zum ersten Abstich festzustellen. Beim Vergleich der Säuren des Mostes und des daraus entstandenen Weines muß bei letzterem die nichtflüchtige Säure in Rechnung 
gezogen werden, nicht aber die Gesamtsäure, da die flüchtigen Säuren erst im Weine meist aus dem Alkohol entstehen und mit der Säure des Mostes in der Regel nichts gemein haben. Die Zahlen der letzten Spalte der Tabelle lehren, daß die Säureabnahme in außerordentlich weiten Grenzen schwankt. Bei den Weißweinen beträgt sie 6,2 bis $47,9 \%$ der ursprünglich im Moste vorhanden gewesenen Menge. Ganz abnorme Verhältnisse zeigen sich bei den Rotweinen. In früheren Jahren wurde hier stets eine sehr starke Säureabnahme festgestellt; dies ist auch bei den 1901-er Weinen teilweise eingetroffen, insofern als bei zwei Rotweinen die Säureabnahme über die Hälfte ihres ursprünglichen Betrages ausmacht. Im Gegensatze hierzu finden wir bei dem Weine No. 53 (aus dem ostdeutschen Weinbaugebiete) nur die geringfügige Säureabnahme des Mostes von 2,7\% verzeichnet. Überhaupt verhalten sich in dieser Hinsicht die 1901-er Weine des ostdeutschen Weinbaugebietes teilweise abnorm. Es sind in der Tabelle zwei Weine enthalten, die genan soviel Gesamtsäure haben, wie der Most gehabt hatte: No. 6 und No. 29, von denen der letztere aus Ostdeutschland stammte. Ferner sind fünf Weine verzeichnet, die mehr Gesamtsäure enthalten als die zugehörigen Moste: No. 30, 31, 32, 47 und 53; sie stammen alle, mit Ausnahme des elsässischen Weines No. 47, aus den ostdeutschen Weinbaugebieten. Dieses ungewöhnliche Verhalten wird offenbar durch den Umstand bedingt, daß die ostdentschen Moste des Jahres 1901 überraschend wenig Säure enthielten; in früheren Jahren, wo die Moste sehr säurereich waren, war die Säureabnahme eine sehr erhebliche.

\title{
Die qualitativen Reaktionen des Wasserstoffsuperoxyds und deren Anwendbarkeit bei Gegenwart von Mileh.
}

\author{
Vou \\ Carl Arnold und Curt Mentzel. \\ Mitteilung aus dem chemischen Institut der Königl. Tierärtlichen \\ Hochschule zu Hannover.
}

Ein neues, zum Nachweise des Wasserstoffsuperoxyds empfehlenswertes Reagens fanden wir in der Vanadinsäure, von der am besten eine Lösung von $1 \mathrm{~g}$ präzipitierter Vanadinsäure in $100 \mathrm{~g}$ verdünnter Schwefelsäure verwendet wird; von dieser gelb gefärbten Lösung setzt man zu je $10 \mathrm{ccm}$ der auf Wasserstoffsuperoxyd zu prüfenden Flüssigkeit 3 Tropfen hinzu; das etwa $4-5$ Gew. $0 \%$ enthaltende käufliche Wasserstoffsuperoxyd gibt mit jedem einfallenden Tropfen dieser Vanadinsäurelösung eine Rotfärbung, die aber im überschüssigen Wasserstoffsuperoxyd verschwindet, indessen auf Zusatz von etwa $1 \mathrm{ccm}$ konzentrierter Salzsäure oder verdünnter Schwefelsäure zu $10 \mathrm{ccm}$ Flüssigkeit wieder erscheint; bei geringem Wasserstoffsuperoxydgehalt genügen 10 Tropfen dieser Säuren. Die Färbung ist in diesem Falle beständig und die Reaktion ebenso scharf, wie die von uns angegebene Benzidin-Cuprisulfatprobe ${ }^{1}$, sie gestattet noch den Nachweis des Wasserstoffsuperoxyds in einer wässerigen $0,0006 \%$-igen Lösung und hat z. B. vor der Reaktion mit Titansäure den Vorzug, daß man rötliche

1) Ber. Deutsch. Chem. Ges. 1902, 35, 1328.

N. 08. 